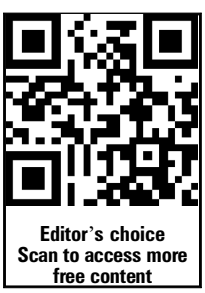

- Additional material is published online only. To view please visit the journal online (http://dx.doi.org/10.1136/ bjsports-2013-093340).

${ }^{1}$ The University of Melbourne, Centre for Health, Exercise \& Sports Medicine, Department of Physiotherapy, School of Health Sciences, Parkville, Victoria, Australia

${ }^{2}$ The University of Queensland, Centre of Clinical Research Excellence in Spinal Pain, Injury \& Health, School of Health \& Rehabilitation Sciences,

St. Lucia, Queensland, Australia

\section{Correspondence to} Dr Rana S Hinman, Department of Physiotherapy, Room 708, Level 7 Alan Gilbert Building, Centre for Health Exercise \& Sports Medicine, School of Health Sciences, The University of Melbourne, VIC 3010, Australia;

ranash@unimelb.edu.au

Accepted 26 August 2014 Published Online First 22 September 2014

\title{
Physical impairments and activity limitations in people with femoroacetabular impingement: a systematic review
}

\author{
Laura E Diamond, ${ }^{1}$ Fiona L Dobson, ${ }^{1}$ Kim L Bennell, ${ }^{1}$ Tim V Wrigley, \\ Paul W Hodges, ${ }^{2}$ Rana S Hinman ${ }^{1}$
}

\section{ABSTRACT}

Background Femoroacetabular impingement (FAl) is a morphological hip condition that can cause hip and/or groin pain in younger active adults. Understanding the nature of physical impairments and activity limitations associated with symptomatic FAl is important to evaluate outcomes and guide development of rehabilitation strategies. The purpose of this systematic review was to establish: (1) whether people with symptomatic FAl demonstrate physical impairments and/or activity limitations compared with people without FAl; and (2) whether treatment affects these parameters.

Methods Four databases (Pubmed, CINAHL, SportDISCUS and Cochrane Library) were searched until the 21 June 2013. Studies evaluated measures of physical impairment and/or activity limitations in people with symptomatic FAl and included either: (1) a comparison control group; or (2) a pretreatment and post-treatment comparison. Methodological quality was assessed using the Newcastle-Ottawa Scale.

Results 16 studies were included. The most commonly reported physical impairment was decreased range of motion (ROM) into directions of hip joint impingement. Other impairments included altered sagittal and frontal plane hip ROM during gait, altered sagittal plane hip ROM during stair climbing, and decreased hip adductor and flexor muscle strength. Effects of surgery on physical impairments are inconsistent but suggest improved hip ROM during gait, but not during stair climbing.

Squatting depth improves following surgical intervention for symptomatic FAI.

Conclusions People with symptomatic FAI demonstrate physical impairments and activity limitations. Surgical intervention may restore some deficiencies, but not all. Further studies of physical impairment and activity limitation are needed to evaluate outcomes from surgical and conservative interventions and to inform rehabilitation programmes.

\section{INTRODUCTION}

Femoroacetabular impingement (FAI) is a morphological hip condition that can cause hip/groin pain and impaired performance in younger active adults. ${ }^{1-4}$ In a 5 -year study of elite athletes who underwent hip arthroscopic surgery, 36\% were treated for FAI. ${ }^{5}$ FAI results from morphological hip abnormalities that abut the proximal femur against the acetabular rim. ${ }^{3}{ }^{6}$ Impingement is caused by abnormal morphology of the femoral head (referred to as cam impingement), excessive acetabular coverage of the femoral head (referred to as pincer impingement) or a combination of the two. ${ }^{346-8}$
Typically, impingement occurs with combined movements of hip flexion, adduction and internal rotation, ${ }^{13}$ though a consistent definition of FAI is lacking in the literature. FAI is associated with sports such as hockey, football and soccer. ${ }^{2} 5910$ FAI can cause symptoms and impair function, and is associated with chondropathy. ${ }^{11}$ The repetitive bony abutment may lead to structural damage, including tearing at the chondrolabral junction, full thickness cartilage delamination and, eventually, hip osteoarthritis. ${ }^{3}{ }^{4} 12$ In sporting populations, recurrent hip pain and increasingly limited range of motion (ROM) secondary to FAI can limit and ultimately compromise athletic performance. ${ }^{9} 1013$

Surgery is the most common treatment for FAI and there is evidence for favourable short-term results of improved function and reduced pain ${ }^{314-16}$; a systematic review reported large within subject effect sizes for follow-ups up to 3 years, ${ }^{16}$ however, another showed outcomes were less favourable in patients with chondropathy. ${ }^{17}$ The long-term clinical results of surgery for FAI, and the role of non-surgical interventions such as rehabilitation and exercise programmes, have not been established. ${ }^{14}$ Non-operative treatments may have a role in managing FAI to alleviate symptoms, potentially resulting in postponement or avoidance of surgery. ${ }^{18}$ The development of rehabilitation strategies to conservatively manage FAI relies not only on patient reported outcomes, but also on an understanding of the associated physical impairments and activity limitations. A clear understanding of these is also necessary to evaluate the effectiveness of surgical intervention.

The physical impairments and activity limitations associated with symptomatic FAI have yet to be synthesised, and systematic reviews of the effects of treatment have largely focused on patient-reported outcomes. ${ }^{1416} 19$ The aim of this study was to systematically appraise the literature to establish: (1) whether people with symptomatic FAI demonstrate physical impairments and/or objectively quantified activity limitations compared with people without FAI; and (2) the effect of treatment on these physical impairments and/or activity limitations.

\section{METHODS}

The systematic review protocol was developed according to guidelines outlined in the PRISMA Statement. ${ }^{20}$

\section{Search strategy}

Electronic searches until the 21 June 2013 were performed by one reviewer in MEDLINE 
(Pubmed), CINAHL and SportDISCUS (EBSCO) and Cochrane Library Databases. Key search terms and synonyms were combined using database-specific truncation terms into three main filters. Variations of the keywords for each filter are summarised as: (1) Population: 'femoracetabular impingement', 'pain', 'injuries', 'hip joint'; (2) Comparison: 'case-control studies', 'case comparison study', 'control', 'normal', 'asymptomatic', 'healthy'; (3) Outcome: 'biomechanics', 'kinetics', 'gait', 'neuromuscular', 'electromyography', 'EMG', 'musculoskeletal', 'range of motion', 'muscle strength', 'impairment', 'motor activity', 'activities of daily living', 'postural balance', 'stair', 'squat', 'joint stiffness', 'muscle weakness', 'pain' (complete search strategy in online supplementary file 1). Studies were limited to the English language and human participants. Supplementary searches of the reference lists of included studies and relevant journals were performed.

\section{Eligibility}

Eligibility was assessed by two independent reviewers (LED and $\mathrm{PN}$ ), with disagreements resolved by consensus or a third reviewer (FD). After removal of duplicates, titles and abstracts were assessed and full texts were obtained for final eligibility screening. Studies were eligible if the population had symptomatic FAI, diagnosed by clinical as well as imaging features. Imaging could include X-ray, MRI or CT scan. All stages and types of FAI were eligible. Studies including people with FAI who had recently undergone a specific intervention (such as arthroscopy or an exercise programme) were eligible if physical impairments and/or activity limitations were objectively measured at preintervention and postintervention time points. Any study design was eligible. Studies were required to report a performance-based measure related to body structure and/or function (ie, impairments such as hip joint biomechanics, neuromuscular activity, strength, ROM and/or objectively quantified activity limitations such as difficulty with squatting, kicking, running, jumping) in accordance with the International Classification of Functioning Disability and Health framework. ${ }^{21}$ Studies including an asymptomatic FAI group, in addition to a symptomatic FAI group, were included if other eligibility requirements were met. Studies were excluded if only those with asymptomatic FAI were evaluated; the full text was not available; the study was not reported in English; or the study reported only patient-reported outcomes.

\section{Quality evaluation}

Methodological quality was assessed by two independent reviewers (LED and FD), with disagreements resolved by consensus. Quality was assessed using the Newcastle-Ottawa Scale (NOS, data online supplementary file 2), a tool designed for cohort and case-control studies, which is reliable and valid for assessing quality of non-randomised studies. ${ }^{22} 23$ Criteria evaluate potential bias based on selection of participants, comparability of study groups and attainment of exposure (case-control studies) or outcome of interest (cohort studies). The NOS uses a star rating system (semi-quantitative) where one star is awarded for each criterion if appropriate methods are reported, with the exception of comparability of cohorts where two stars are awarded if a study controls for more than one comparison factor. The scale ranges from zero to nine stars. ${ }^{24}$ The casecontrol scale and cohort study scale were used to evaluate included cross-sectional and intervention studies, respectively. Levels of evidence were assessed using the Centre for Evidence Based Medicine (CEBM) scale. ${ }^{25}$

\section{Data extraction}

A single reviewer (LED) extracted all data, including study design, recruitment source, sample size, population characteristics, intervention details, comparison group characteristics and any confounding factor controlled for. For mixed design studies (eg, studies with pre intervention and postintervention measures of an FAI group as well as an asymptomatic cohort), the preintervention data were extracted for cross-sectional comparison with asymptomatic controls. Quantitative data relating to physical impairment and/or activity limitation were extracted. For studies evaluating ROM and joint forces (which typically reported a range of variables), only the main and/or significant findings were extracted.

\section{Statistical analysis}

Percentage agreement and the $\kappa$ statistic (95\% CI) were used to provide absolute agreement and an estimate of level of agreement between raters when scoring the methodological quality of the included studies. Within-in subject effect sizes and 95\% CIs were calculated for intervention studies using Hedges' $g$ (average). This method is recommended for correlated groups used in meta-analysis and is useful when the number of paired comparisons is small. ${ }^{26} 27$

\section{RESULTS}

The search identified 1724 articles (figure 1), of which 16 studies were eligible. A description of the 14 cross-sectional comparisons is provided in table 1 . Table 2 provides descriptive data for the five preintervention and postintervention studies (three of which included a control group for comparison and thus are also included in table 1). Mean age of participants ranged from 24.7 to 35.5 years. The proportion of men ranged from $36 \%$ to $100 \%$, with 14 studies having a predominantly male sample. ${ }^{18} 28-40$

\section{Cross-sectional comparisons of people with and without symptomatic FAl}

Study quality was moderate to high, with scores ranging from 5 to 8 stars (figure 2). Studies with control groups not imaged to ensure no evidence of FAI did not earn a star for selection (definition of controls) and exposure (control group). All included cross-sectional studies were rated as level 4 evidence (figure 2).

The inter-rater agreement for methodological quality of studies was excellent (absolute agreement $=95 \%, \kappa=0.92,95 \%$ CI 0.87 to 0.97 ). All disagreements were easily resolved using a consensus method between the two raters.

Table 3 summarises the main cross-sectional findings. Eleven of the 14 studies included a comparison group of age-matched and gender-matched controls. ${ }^{28-35} 40-42$ Two used historic controls comprised of the contralateral hips of older total hip replacement patients, ${ }^{36} 37$ and one used the contralateral limbs of participants with symptomatic FAI. ${ }^{18}$ Five studies did not use imaging to ensure no evidence of FAI in the asymptomatic control group. $^{34} 35$ 40-42

Twelve of 14 studies evaluated hip ROM; three using CT 323637 and six using 3-dimensional motion analysis. ${ }^{28-30} \quad 33-35$ Other physical impairments were hip muscle strength, ${ }^{41}$ fatigue $^{42}$ and neuromuscular activity. ${ }^{41}{ }^{42}$ The only objectively quantified activity limitation was squatting. ${ }^{30}$ A meta-analysis was not performed because of the small number of studies and high heterogeneity in outcome measures. 

selection process.
Figure 1 Flow chart of study

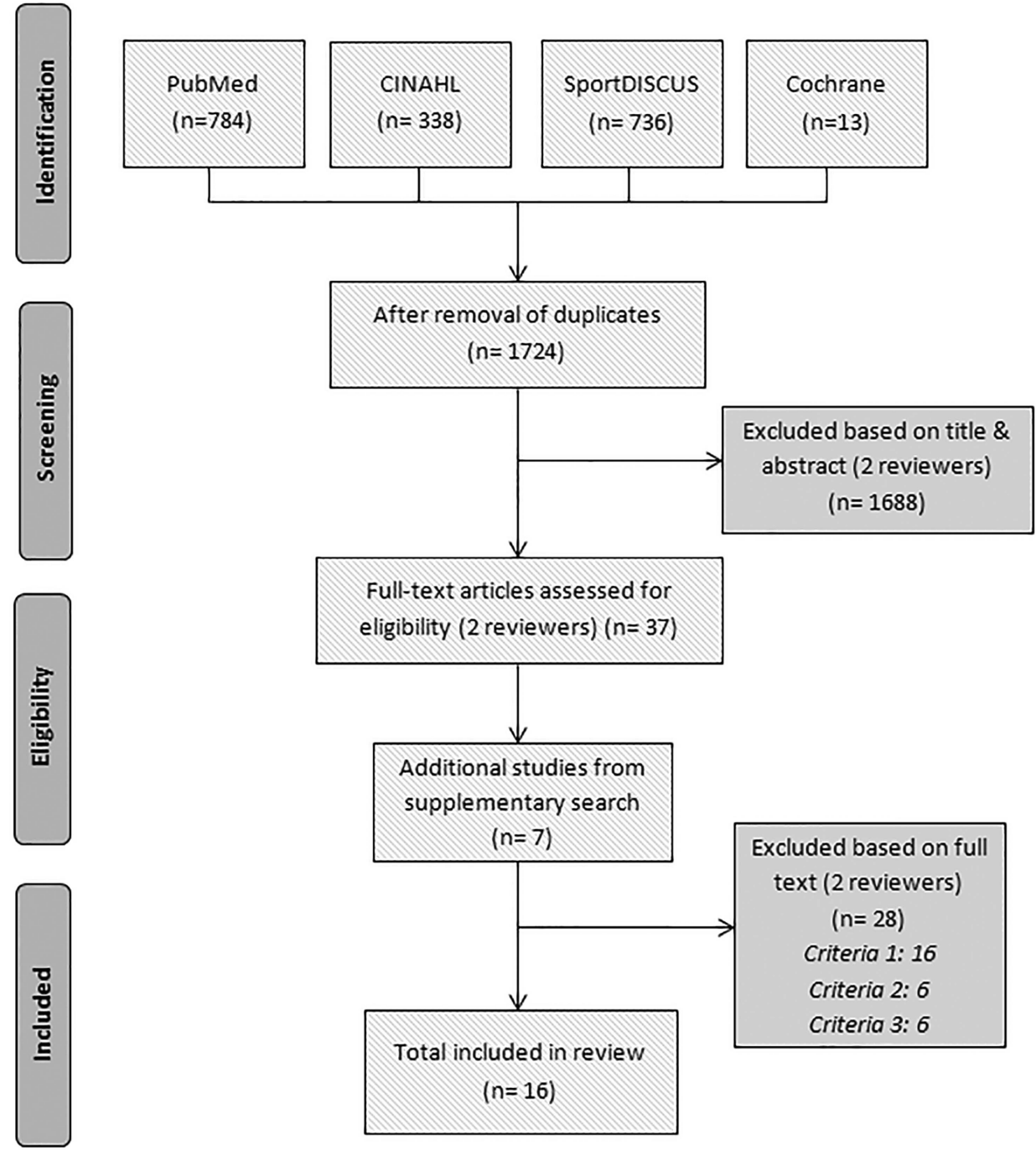

ROM-compared to controls with no FAI hip morphology

Two studies simulated hip ROM using CT and found that people with symptomatic FAI had less ROM towards positions of impingement (flexion/internal rotation in $90^{\circ}$ flexion) and abduction $^{36} 37$ than an older control group. Two further studies examined passive hip ROM in symptomatic FAI compared with asymptomatic FAI and a control group without FAI. ${ }^{31} 32$ The first used electromagnetic tracking to measure hip ROM and found the symptomatic FAI group exhibited significantly less flexion, external rotation and internal rotation in $90^{\circ}$ hip flexion compared with both control groups. ${ }^{31}$ Similarly, the second study, using CT, reported that the symptomatic FAI group exhibited significantly decreased range of internal rotation in hip flexion and significantly decreased range of internal rotation during an impingement test than the control groups. The asymptomatic FAI group also had significantly lower values for these tests than the control group. ${ }^{32}$ Similarly, a study using three-dimensional motion analysis during a series of maximum dynamic hip ROM trials, reported the symptomatic FAI group used significantly less total hip ROM in the sagittal plane and significantly less hip ROM into peak hip abduction, and internal rotation in $90^{\circ}$ hip flexion and external rotation in $90^{\circ}$ hip flexion, compared with a control group. ${ }^{28}$ Hip ROM values were similarly less in a different study when the symptomatic FAI limb was compared with the contralateral asymptomatic limb, but whether differences were statistically significant was not reported. ${ }^{18}$

Two studies compared dynamic hip ROM during gait in people with symptomatic FAI to controls using threedimensional motion analysis. ${ }^{29} 33$ Both reported significantly decreased frontal plane hip ROM (adduction-abduction) in symptomatic FAI, ${ }^{29} 33$ and one reported significantly decreased sagittal hip ROM (flexion-extension). ${ }^{33}$

One study evaluated dynamic ROM during squatting using three-dimensional motion analysis and found that the symptomatic FAI group used significantly less sagittal plane pelvic ROM than controls. ${ }^{30}$

ROM-compared to controls with uncertain hip morphology

A measurement study comparing a goniometer and an electromagnetic tracking system reported significantly less passive hip abduction in a symptomatic FAI group compared with a control group. ${ }^{40}$

Two studies compared dynamic hip ROM during gait in people with symptomatic FAI to controls using threedimensional motion analysis. ${ }^{34} 35$ Both reported significant decreased frontal plane hip ROM (adduction-abduction) in symptomatic FAI. ${ }^{34}{ }^{35}$ One reported significantly decreased sagittal hip ROM (flexion-extension), ${ }^{35}$ while similarly, the second reported significantly decreased peak hip extension in 
Cross-sectional studies

\begin{tabular}{|c|c|c|c|c|c|}
\hline \multirow[b]{2}{*}{ Author, Year } & \multirow[b]{2}{*}{ FAI group } & \multicolumn{2}{|l|}{ Control group } & \multirow{2}{*}{$\begin{array}{l}\text { Comparability } \\
\text { between groups* }\end{array}$} & \multirow{2}{*}{$\begin{array}{l}\text { Impairment and/or activity } \\
\text { limitation investigated }\end{array}$} \\
\hline & & Asymptomatic with no FAI & Asymptomatic FAl & & \\
\hline Tannast, 2007 & $\begin{array}{l}\mathrm{n}=31 \text { (all types FAI) } \\
27(87 \%) \text { males } \\
\text { Mean } \pm \text { SD age }=31.1 \pm 9.4 \text { years } \\
\text { Recruited from outpatient clinic } \\
\text { Diagnosis based on: } \\
\text { Radiographic criteria (not specified) } \\
\text { Clinical criteria (not specified) }\end{array}$ & $\begin{array}{l}\mathrm{n}=36 \\
23(64 \%) \text { males } \\
\text { Mean } \pm \mathrm{SD} \text { age }=53.7 \pm 11.3 \text { years } \\
\text { Recruited from contralateral hip THR patients } \\
\text { Exclusion based on: } \\
\text { - Radiographic } \mathrm{OA}>\text { grade } 1 \\
\text { - Centre edge angle }<25^{\circ} \\
\text { CT measurements }\left(\alpha \text { angle }<50^{\circ} \text {; femoral retroversion) }\right. \\
\text { - Symptomatic (history of hip pain) }\end{array}$ & & None & ROM \\
\hline Kubiak-Langer, 2007 & $\begin{array}{l}\mathrm{n}=28 \text { (all types FAl) } \\
24(86 \%) \text { males } \\
\text { Mean } \pm \mathrm{SD} \text { age }=35.4 \pm 10.4 \text { years } \\
\text { Recruited from outpatient clinic } \\
\text { Diagnosis based on: } \\
\text { - Radiographic criteria (not specified) } \\
\text { Physical findings (positive impingement test) } \\
\text { - Symptoms (not specified) }\end{array}$ & $\begin{array}{l}\mathrm{n}=33 \\
20(61 \%) \text { males } \\
\text { Mean } \pm \mathrm{SD} \text { age }=53 \pm 11.1 \text { years } \\
\text { Recruited from contralateral hip THR patients } \\
\text { Exclusion based on: } \\
\text { Radiographic } \mathrm{OA}>\text { grade } 1 \\
\text { Centre edge angle }<25^{\circ} \\
\text { - CT measurements }\left(\alpha \text { angle }<50^{\circ} \text {; femoral retroversion) }\right. \\
\text { - Symptomatic (history of hip pain, or hip surgery) }\end{array}$ & & None & ROM \\
\hline Kennedy, 2009a & $\begin{array}{l}\mathrm{n}=17(\mathrm{Cam} \text { FAI) } \\
10(59 \%) \text { males } \\
\text { Mean } \pm \mathrm{SD} \text { age }=35.5 \pm 10.6 \text { years } \\
\text { Recruited from NR } \\
\text { Diagnosis based on: } \\
\text { Radiographs (A/P and Dunn view) (mean } \alpha \text { angle } \\
\left.>50.5^{\circ}\right) \\
\text { - Positive impingement test }\end{array}$ & $\begin{array}{l}\mathrm{n}=14 \\
8(57 \%) \text { males } \\
\text { Mean } \pm \mathrm{SD} \text { age }=34.2 \pm 9.5 \text { years } \\
\text { Recruited from NR } \\
\text { Inclusion based on: } \\
\text { Radiographs (A/P and Dunn view) (spherical femoral } \\
\quad \text { heads) } \\
\text { - Asymptomatic (no history of lower limb injury) }\end{array}$ & & $\begin{array}{l}\text { Age } \\
\text { Gender } \\
\text { BMI }\end{array}$ & ROM \\
\hline Kennedy, 2009b & $\begin{array}{l}\mathrm{n}=17(\text { Cam FAI) } \\
10(59 \%) \text { males } \\
\text { Mean } \pm \text { SD age }=35.5 \pm 10.6 \text { years } \\
\text { Recruited from NR } \\
\text { Diagnosis based on: } \\
\text { Radiographs (A/P and Dunn view) (mean } \alpha \text { angle } \\
>50.5^{\circ} \text { ) } \\
\text { Dositive impingement test }\end{array}$ & $\begin{array}{l}\mathrm{n}=14 \\
8(57 \%) \text { males } \\
\text { Mean } \pm \mathrm{SD} \text { age }=34.2 \pm 9.5 \text { years } \\
\text { Recruited from NR } \\
\text { Inclusion based on: } \\
\text { - Radiographs (A/P and Dunn view) (spherical femoral } \\
\text { heads) } \\
\text { Asymptomatic (no history of lower limb injury, no hip } \\
\text { pain) }\end{array}$ & & $\begin{array}{l}\text { Age } \\
\text { Gender } \\
\text { BMI }\end{array}$ & ROM \\
\hline Lamontagne, 2009 & $\begin{array}{l}\mathrm{n}=15(\mathrm{Cam} \text { FAl) } \\
9(60 \%) \text { males } \\
\text { Mean } \pm \text { SD age }=35.3 \pm 9.1 \text { years } \\
\text { Recruited from NR } \\
\text { Diagnosis based on: } \\
\text { Radiographs (A/P and Dunn view) (mean } \alpha \text { angle } \\
\left.>50.5^{\circ}\right) \\
\text { Positive impingement test }\end{array}$ & $\begin{array}{l}\mathrm{n}=11 \\
6(55 \%) \text { males } \\
\text { Mean } \pm \mathrm{SD} \text { age }=34.5 \pm 10.1 \text { years } \\
\text { Recruited from NR } \\
\text { Inclusion based on: } \\
\text { - Radiographs (A/P and Dunn view) (spherical femoral } \\
\text { heads) } \\
\text { - Asymptomatic (no history of lower limb injury) }\end{array}$ & & $\begin{array}{l}\text { Age } \\
\text { Gender } \\
\text { BMI }\end{array}$ & $\begin{array}{l}\text { Squatting ability } \\
\text { ROM }\end{array}$ \\
\hline Nussbaumer, 2010 & $\begin{array}{l}\mathrm{n}=15(\text { all types FAl }) \\
8(53 \%) \text { males } \\
\text { Mean } \pm \mathrm{SD} \text { age }=35 \pm 11 \text { years }\end{array}$ & $\begin{array}{l}\mathrm{n}=15 \\
8(53 \%) \text { males } \\
\text { Mean } \pm \mathrm{SD} \text { age }=34 \pm 10 \text { years }\end{array}$ & & $\begin{array}{l}\text { Age } \\
\text { Gender }\end{array}$ & ROM \\
\hline
\end{tabular}




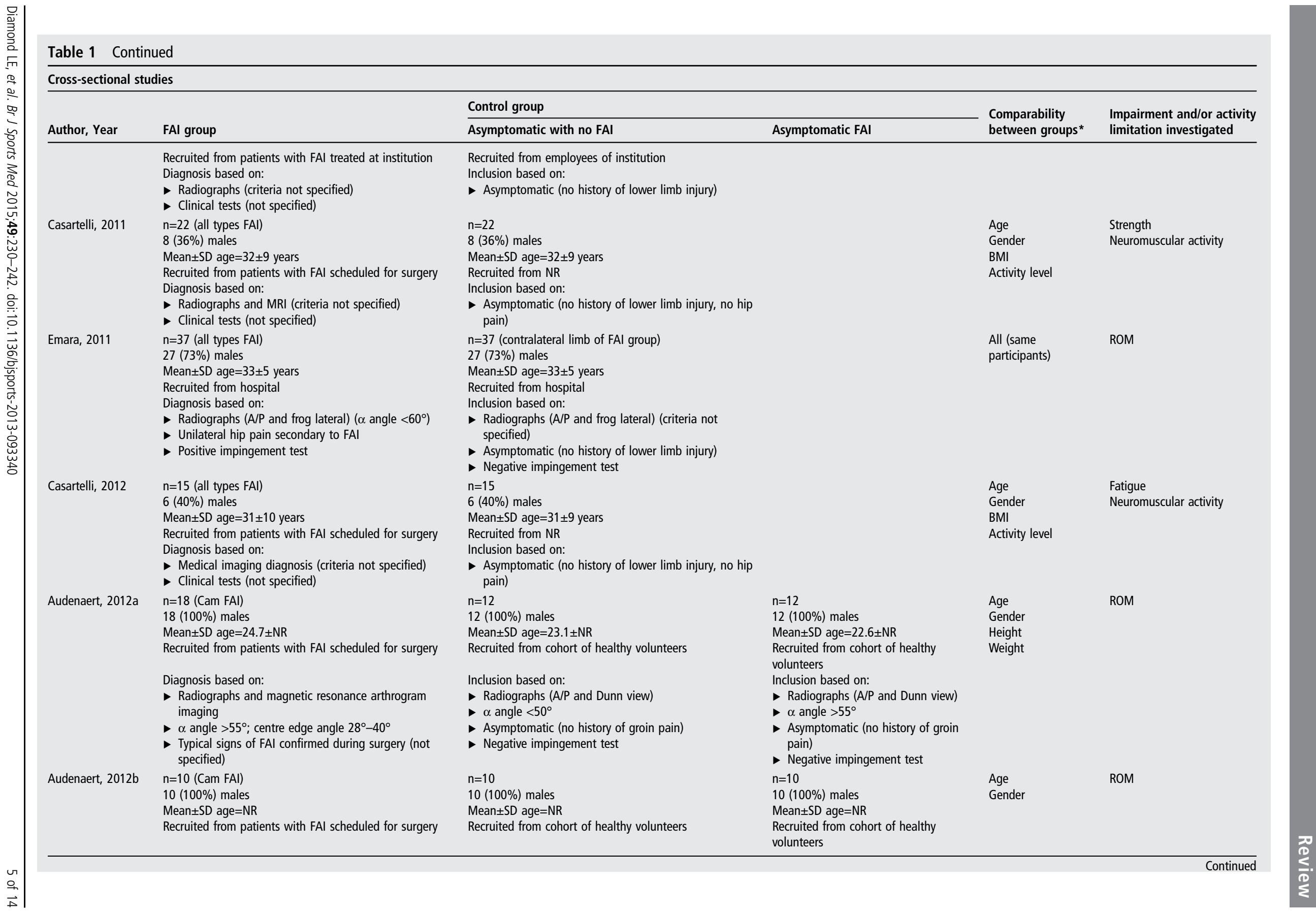


Table 1 Continued

Cross-sectional studies

\begin{tabular}{|c|c|c|c|c|c|}
\hline \multirow[b]{2}{*}{ Author, Year } & \multirow[b]{2}{*}{ FAl group } & \multicolumn{2}{|l|}{ Control group } & \multirow{2}{*}{$\begin{array}{l}\text { Comparability } \\
\text { between groups* }\end{array}$} & \multirow{2}{*}{$\begin{array}{l}\text { Impairment and/or activity } \\
\text { limitation investigated }\end{array}$} \\
\hline & & Asymptomatic with no FAl & Asymptomatic FAI & & \\
\hline & $\begin{array}{l}\text { Diagnosis based on: } \\
\text { Radiographs } \\
\quad \alpha \text { angle }>55^{\circ} \text {; centre edge angle } 28^{\circ}-40^{\circ} \\
\text { Typical signs of FAl confirmed with a CT scan }\end{array}$ & $\begin{array}{l}\text { Inclusion based on: } \\
\text { Radiographs (A/P and Dunn view) } \\
\alpha \text { angle }<50^{\circ} \\
\text { Asymptomatic (no history of groin pain) } \\
\text { - Negative impingement test }\end{array}$ & $\begin{array}{l}\text { Inclusion based on: } \\
\text { Radiographs (A/P and Dunn view) } \\
\quad \alpha \text { angle }>55^{\circ} \\
\text { Asymptomatic (no history of groin } \\
\text { pain) } \\
\text { Negative impingement test }\end{array}$ & & \\
\hline Brisson, 2013 & $\begin{array}{l}\mathrm{n}=10(\text { Cam FAl }) \\
7(70 \%) \text { males } \\
\text { Mean } \pm \mathrm{SD} \text { age }=29.9 \pm 7.2 \text { years } \\
\text { Recruited from NR } \\
\text { Diagnosis based on: } \\
- \text { Radiographs (A/P and Dunn view) (mean } \alpha \text { angle } \\
\left.\quad>50.5^{\circ}\right) \\
- \text { Positive impingement test }\end{array}$ & $\begin{array}{l}\mathrm{n}=13 \\
8(62 \%) \text { males } \\
\text { Mean } \pm \mathrm{SD} \text { age }=34.2 \pm 9.9 \text { years } \\
\text { Recruited from NR } \\
\text { Inclusion based on: } \\
\text { - Radiographs (A/P and Dunn view) (spherical femoral } \\
\text { heads) } \\
\text { pasymptomatic (no history of lower limb injury, no hip } \\
\text { pain) }\end{array}$ & & $\begin{array}{l}\text { Age } \\
\text { Gender } \\
\text { BMI }\end{array}$ & ROM \\
\hline Hunt, 2013 & $\begin{array}{l}\mathrm{n}=30 \text { (all types FAl) } \\
25(83 \%) \text { males } \\
\text { Mean } \pm \mathrm{SD} \text { age }=35.5 \pm 10.6 \text { years } \\
\text { Recruited from orthopaedic surgery } \\
\text { Diagnosis based on: } \\
\text { Radiographs (A/P) (Pincer FAl: positive cross-over) } \\
\text { MR arthrogram imaging (cam FAl: } \alpha \text { angle) } \\
\text { - Positive impingement signs } \\
\text { - Presence of anterior groin pain }\end{array}$ & $\begin{array}{l}\mathrm{n}=30 \\
20(67 \%) \text { males } \\
\text { Mean } \pm \mathrm{SD} \text { age }=34.2 \pm 9.5 \text { years } \\
\text { Recruited from university community } \\
\text { Inclusion based on: } \\
\text { Asymptomatic (no history of lower limb injury, no hip } \\
\text { pain) } \\
\text { - Negative impingement test }\end{array}$ & & $\begin{array}{l}\text { Age } \\
\text { Gender }\end{array}$ & ROM \\
\hline Rylander, 2013 & $\begin{array}{l}\mathrm{n}=17 \text { (all types FAI) } \\
12(71 \%) \text { males } \\
\text { Mean } \pm \mathrm{SD} \text { age }=35.4 \pm 8.9 \text { years } \\
\text { Recruited from patients with FAI scheduled for surgery } \\
\text { Diagnosis based on: } \\
\text { Radiographs and MRI } \\
\text { Cam: mean } \alpha \text { angle }>54^{\circ} \text {; Pincer: centre edge angle } \\
>35^{\circ} \\
\text { - History and clinical examination (criteria not specified) }\end{array}$ & $\begin{array}{l}\mathrm{n}=17 \\
12(71 \%) \text { males } \\
\text { Mean } \pm \mathrm{SD} \text { age }=34.9 \pm 9.7 \text { years } \\
\text { Recruited from community } \\
\text { Inclusion based on: } \\
\text { Asymptomatic (no history of lower limb injury, no hip } \\
\text { pain) }\end{array}$ & & $\begin{array}{l}\text { Age } \\
\text { Gender } \\
\text { BMI }\end{array}$ & ROM \\
\hline
\end{tabular}

${ }^{*}$ Features named as those that the groups were comparable on.

A $/ P$, anteroposterior; BMI, body mass index; FAI, femoroacetabular impingement; NR, not reported; OA, osteoarthritis; ROM, range of motion; THP, total hip replacement. 


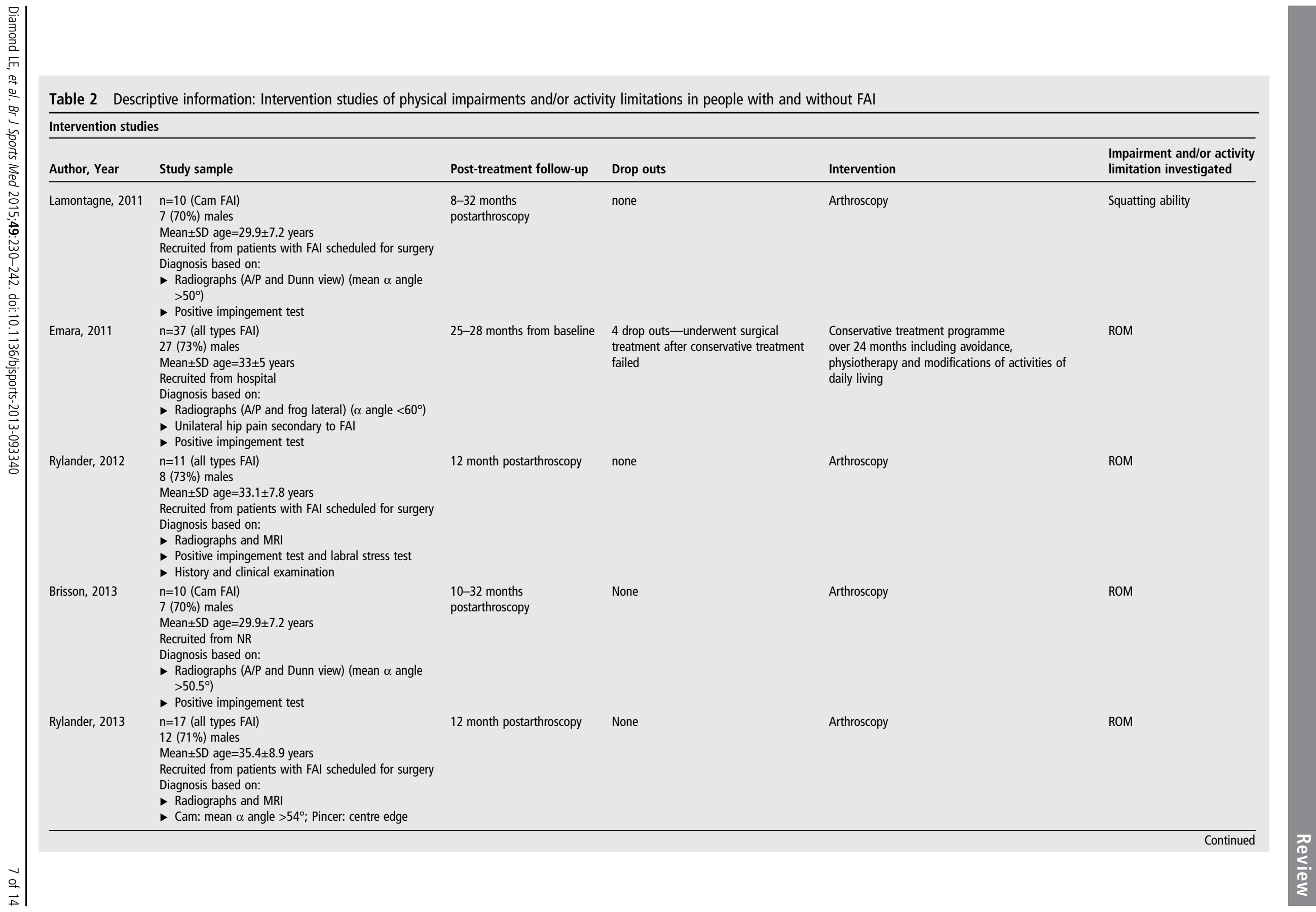


Figure 2 Quality evaluation of studies using the Newcastle-Ottawa scale.

\begin{tabular}{|c|c|c|c|c|c|}
\hline Author, Year & Selection & Compar ability & Exposure & Total Stars & $\begin{array}{l}\text { Level of } \\
\text { Evidence }\end{array}$ \\
\hline Tannast, 2007 & 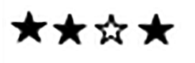 & 论络 & 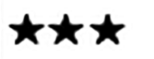 & 6 & 4 \\
\hline Kubiak-Langer, 2007 & ‘丈々九 & 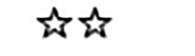 & 大丈t & 6 & 4 \\
\hline Kennedy, 20093 & 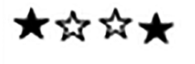 & $\star \star$ & 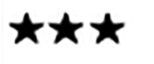 & 7 & 4 \\
\hline Kennedy, 2009\% & 必次太 & $\star \star$ & 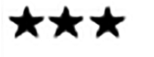 & 7 & 4 \\
\hline Lamontagne, 2009 & 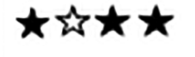 & $\star \star$ & 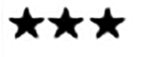 & 8 & 4 \\
\hline Nussbaumer, 2010 & 太方九次 & $\star \star$ & 大次九 & 0 & 4 \\
\hline Casartelli, 2011 & 大地方公 & $\star \star$ & $\star \Delta \star \Delta$ & 5 & 4 \\
\hline Emara, 2011 $1^{x}$ & えれ公え & $\star \star \star$ & 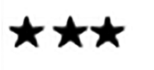 & 8 & 4 \\
\hline Casartelli, 2012 & 必地 & $\star \star \star$ & 大勀 & 5 & 4 \\
\hline A udenaert, $2012 \mathrm{~s}$ & 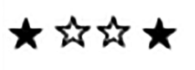 & $\star \star \star$ & 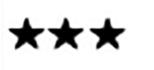 & 7 & 4 \\
\hline A udenaert, 2012b & 大公的大 & $\star \star$ & 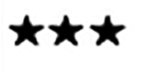 & 7 & 4 \\
\hline Brisson, 2013^ & 头论太 & $\star \star \star x$ & $\star x \star x$ & 7 & 4 \\
\hline Hunt, 2013 & 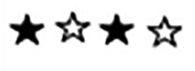 & $\star \star$ & 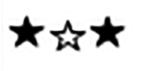 & 6 & 4 \\
\hline Rylander. $2013^{x}$ & 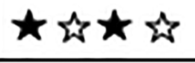 & $\star x$ & 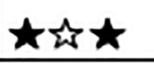 & 0 & 4 \\
\hline
\end{tabular}

B

\begin{tabular}{|c|c|c|c|c|c|}
\hline Author, Year & Selection & Compar ability & Outcome & Total Stars & $\begin{array}{c}\text { Level of } \\
\text { Evidence }\end{array}$ \\
\hline Lamontagne, 2011 & 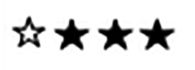 & $\star \star$ & 车公 & 7 & $3 b$ \\
\hline Emars, 2011 & 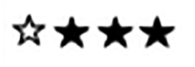 & $\star \star \star$ & 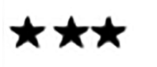 & 8 & $3 b$ \\
\hline Rylander, 2012 & 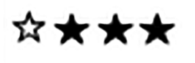 & $\star \star \star$ & $\star \star \star x \star \Delta$ & 8 & 36 \\
\hline 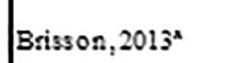 & 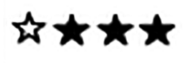 & $\star \star x$ & 车公太 & 7 & $3 b$ \\
\hline Rylander, $2013^{*}$ & $\Delta \star \Delta \star \Delta$ & $\star \star x$ & $x \star x$ & 8 & $3 b$ \\
\hline
\end{tabular}

symptomatic FAI. $^{34}$ Both studies demonstrated significantly reduced peak hip internal rotation ROM in symptomatic FAI. ${ }^{34} 35$

One study compared dynamic ROM in people with symptomatic FAI to a control group during stair climbing using threedimensional motion analysis. ${ }^{35}$ Significantly reduced sagittal plane hip ROM, plus decreased maximum hip extension and internal rotation ROM were observed with FAI.

\section{Joint moments}

Three studies evaluated net hip joint moments during gait. ${ }^{29} 3334$ Only one reported decreased peak hip flexion and extension moments in people with symptomatic FAI, ${ }^{34}$ but used a control group not imaged to ensure no evidence of FAI. The other two studies found no differences when controls were imaged to ensure no FAI. ${ }^{29} 33$
Muscle strength and fatigue-compared with controls with uncertain hip morphology

One study evaluated muscle strength using hand-held isometric and isokinetic dynamometry. ${ }^{41}$ Decreased isometric maximum voluntary torque normalised to body mass of hip adductor, abductor and flexor and external rotator muscles was observed with symptomatic FAI compared with a control group. ${ }^{41}$ Hip internal rotators and extensors strength was not significantly different between groups. ${ }^{41}$ In a separate study, the same authors evaluated submaximal isometric fatigue (torque fluctuations and electromyography (EMG) magnitude changes) and maximal isokinetic fatigue (rate of torque decline) of hip flexors. While hip flexor strength relative to body mass was $21 \%$ lower in symptomatic FAI compared with control participants, there were no significant differences between groups in any of the fatigue indices. $^{42}$ 


\begin{tabular}{|c|c|c|c|c|c|c|}
\hline \multicolumn{7}{|c|}{ Cross-sectional studies } \\
\hline \multirow[b]{2}{*}{ Author, Year } & \multirow{2}{*}{$\begin{array}{l}\text { Impairment and/or } \\
\text { activity limitation } \\
\text { investigated }\end{array}$} & \multirow[b]{2}{*}{ Measurement method } & \multirow[b]{2}{*}{ Selected variables measured } & \multirow[b]{2}{*}{$\begin{array}{l}\text { FAl group } \\
(\text { mean } \pm S D)\end{array}$} & \multicolumn{2}{|c|}{ Control group $(m e a n \pm S D)$} \\
\hline & & & & & $\begin{array}{l}\text { Asymptomatic } \\
\text { with no FAl }\end{array}$ & $\begin{array}{l}\text { Asymptomatic } \\
\text { FAI }\end{array}$ \\
\hline Tannast, 2007 & ROM & CT modelling & $\begin{array}{l}\text { Hip flexion }\left({ }^{\circ}\right) \\
\text { Hip internal rotation in } 90^{\circ} \text { flexion }\left({ }^{\circ}\right) \\
\text { Hip abduction }\left(^{\circ}\right)\end{array}$ & $\begin{array}{l}105 \pm 16.1^{*} \\
11.7 \pm 7.1^{*} \\
51.9 \pm 12.1^{*}\end{array}$ & $\begin{array}{l}121 \pm 11.8 \\
35 \pm 12 \\
63 \pm 11.1\end{array}$ & \\
\hline Kubiak-Langer, 2007 & ROM & CT modelling & $\begin{array}{l}\text { Hip flexion }\left(^{\circ}\right) \\
\text { Hip internal rotation in } 90^{\circ} \text { flexion }\left(^{\circ}\right) \\
\text { Hip abduction }\left({ }^{\circ}\right)\end{array}$ & $\begin{array}{l}105.2 \pm 12.2^{*} \\
11.1 \pm 6.9^{*} \\
51.7 \pm 12.2^{*}\end{array}$ & $\begin{array}{l}122 \pm 16.3 \\
35.2 \pm 6.9 \\
63.3 \pm 10.9\end{array}$ & \\
\hline Kennedy, 2009a & ROM & $\begin{array}{l}\text { Three-dimensional kinematics of } \\
\text { dynamic ROM }\end{array}$ & $\begin{array}{l}\text { Sagittal hip ROM }\left({ }^{\circ}\right) \\
\text { Peak hip abduction angle }\left({ }^{\circ}\right) \\
\text { Peak hip internal rotation in } 90^{\circ} \text { flexion }\left({ }^{\circ}\right) \\
\text { Peak hip external rotation in } 90^{\circ} \text { flexion }\left({ }^{\circ}\right) \\
\text { Total transverse hip ROM in } 90^{\circ} \text { flexion }\left(^{\circ}\right)\end{array}$ & $\begin{array}{l}130.9 \pm 13.5 \dagger \\
38.3 \pm 9.1^{*} \\
7.9 \pm 3.4^{*} \\
20.3 \pm 4.5^{*} \\
28.2 \pm 6.7^{*}\end{array}$ & $\begin{array}{l}140.5 \pm 9.0 \\
47.5 \pm 5.7 \\
12.4 \pm 4.8 \\
26.0 \pm 7.0 \\
38.5 \pm 9.7\end{array}$ & \\
\hline Kennedy, 2009b & ROM & $\begin{array}{l}\text { Three-dimensional motion analysis } \\
\text { during gait }\end{array}$ & $\begin{array}{l}\text { Peak hip abduction angle }\left(^{\circ}\right) \\
\text { Frontal hip ROM }\left(^{\circ}\right) \\
\text { Pelvic roll }\left({ }^{\circ}\right)\end{array}$ & $\begin{array}{l}\mathrm{NR}^{*} \text { (decrease) } \\
\mathrm{NR}^{*} \text { (decrease) } \\
\mathrm{NR}^{*} \text { (decrease) }\end{array}$ & $\begin{array}{l}\text { NR } \\
\text { NR } \\
\text { NR }\end{array}$ & \\
\hline Lamontagne, 2009 & $\begin{array}{l}\text { Squatting ability } \\
\text { ROM }\end{array}$ & $\begin{array}{l}\text { Three-dimensional motion analysis } \\
\text { during squatting }\end{array}$ & $\begin{array}{l}\text { Mean max squat depth (\% leg length) } \\
\text { Sagittal pelvic ROM }\left(^{\circ}\right)\end{array}$ & $\begin{array}{l}41.5 \pm 12.5 \dagger \\
14.7 \pm 8.4^{*}\end{array}$ & $\begin{array}{l}32.3 \pm 6.8 \\
24.2 \pm 6.8\end{array}$ & \\
\hline Nussbaumer, 2010 & ROM & $\begin{array}{l}\text { Goniometer } \\
\text { Electromagnetic tracking system }\end{array}$ & $\begin{array}{l}\text { Hip abduction }\left({ }^{\circ}\right) \\
\text { Hip abduction }\left({ }^{\circ}\right)\end{array}$ & $\begin{array}{l}30.4 \pm 7.3^{*} \\
28.5 \pm 6.7^{*}\end{array}$ & $\begin{array}{l}39.3 \pm 7.4 \\
37.3 \pm 8.0\end{array}$ & \\
\hline Casartelli, 2011 & $\begin{array}{l}\text { Neuromuscular } \\
\text { activity }\end{array}$ & $\begin{array}{l}\text { Isometric maximal voluntary contraction } \\
\text { via dynamometer }\end{array}$ & $\begin{array}{l}\text { Hip adduction }(\mathrm{Nm} / \mathrm{kg}) \\
\text { Hip abduction }(\mathrm{Nm} / \mathrm{kg}) \\
\text { Hip internal rotation }(\mathrm{Nm} / \mathrm{kg}) \\
\text { Hip external rotation }(\mathrm{Nm} / \mathrm{kg}) \\
\text { Hip flexion }(\mathrm{Nm} / \mathrm{kg}) \\
\text { Hip extension }(\mathrm{Nm} / \mathrm{kg}) \\
\text { RF activity level }(\mu \mathrm{V}) \\
\text { TFL activity level }(\mu \mathrm{V})\end{array}$ & $\begin{array}{l}1.57 \pm 0.82^{*} \\
1.81 \pm 0.43 \dagger \\
0.47 \pm 0.16 \\
0.46 \pm 0.21 \dagger \\
0.87 \pm 0.46^{*} \\
1.64 \pm 1.00 \\
186 \pm 131 \\
401 \pm 251 \dagger\end{array}$ & $\begin{array}{l}2.17 \pm 0.49 \\
2.03 \pm 0.31 \\
0.55 \pm 0.17 \\
0.56 \pm 0.15 \\
1.17 \pm 0.37 \\
1.66 \pm 0.86 \\
294 \pm 184 \\
582 \pm 323\end{array}$ & \\
\hline Emara, 2011 & ROM & Not reported & $\begin{array}{l}\text { Hip flexion }\left(^{\circ}\right) \\
\text { Hip extension }\left(^{\circ}\right) \\
\text { Hip abduction }\left({ }^{\circ}\right) \\
\text { Hip adduction }\left(^{\circ}\right) \\
\text { Hip external rotation in flexion }\left(^{\circ}\right) \\
\text { Hip external rotation in extension }\left({ }^{\circ}\right) \\
\text { Hip internal rotation in flexion }\left(^{\circ}\right) \\
\text { Hip internal rotation in extension }\left({ }^{\circ}\right)\end{array}$ & $\begin{array}{l}95.0 \pm 0.4 \\
4.0 \pm 1.6 \\
37.0 \pm 0.4 \\
17.0 \pm 7.0 \\
28.5 \pm 0.5 \\
25.3 \pm 0.3 \\
9.4 \pm 0.3 \\
15.8 \pm 0.4\end{array}$ & $\begin{array}{l}\ddagger 103.0 \pm 2.6 \S \\
4.3 \pm 1.7 \S \\
43.0 \pm 3.3 \S \\
19.0 \pm 8.0 \S \\
33.9 \pm 4.0 \S \\
29.7 \pm 3.2 \S \\
14.6 \pm 2.9 \S \\
19.0 \pm 2.6 \S\end{array}$ & \\
\hline Casartelli, 2012 & Fatigue & $\begin{array}{l}\text { Submaximal isometric fatigue via } \\
\text { dynamometer }\end{array}$ & Max isometric torque hip flexors $(\mathrm{Nm} / \mathrm{kg})$ & $0.96 \pm 0.46 \dagger$ & $1.21 \pm 0.38$ & \\
\hline & & $\begin{array}{l}\text { Maximal isokinetic fatigue via } \\
\text { dynamometer }\end{array}$ & Max isokinetic torque hip flexors $(\mathrm{Nm} / \mathrm{kg})$ & $0.97 \pm 0.38 \dagger$ & $1.16 \pm 0.36$ & \\
\hline & $\begin{array}{l}\text { Neuromuscular } \\
\text { activity }\end{array}$ & $\begin{array}{l}\text { Muscle activation of the RF and TFL } \\
\text { during submaximal isometric fatigue via } \\
\text { surface electromyography }\end{array}$ & RF and TFL activity level $(\mu \mathrm{V})$ & Not significant & Not significant & \\
\hline
\end{tabular}


Table 3 Continued

Cross-sectional studies

\begin{tabular}{|c|c|c|c|c|c|c|}
\hline \multirow[b]{2}{*}{ Author, Year } & \multirow{2}{*}{$\begin{array}{l}\text { Impairment and/or } \\
\text { activity limitation } \\
\text { investigated }\end{array}$} & \multirow[b]{2}{*}{ Measurement method } & \multirow[b]{2}{*}{ Selected variables measured } & \multirow[b]{2}{*}{$\begin{array}{l}\text { FAl group } \\
(m e a n \pm S D)\end{array}$} & \multicolumn{2}{|c|}{ Control group (mean $\pm S D$ ) } \\
\hline & & & & & $\begin{array}{l}\text { Asymptomatic } \\
\text { with no FAl }\end{array}$ & $\begin{array}{l}\text { Asymptomatic } \\
\text { FAI }\end{array}$ \\
\hline Audenaert, 2012a & ROM & $\begin{array}{l}\text { Electromagnetic tracking system to measure } \\
\text { kinematics }\end{array}$ & $\begin{array}{l}\text { Neutral hip internal rotation }\left(^{\circ}\right) \\
\text { Neutral hip external rotation }\left(^{\circ}\right) \\
\text { Hip flexion }\left({ }^{\circ}\right) \\
\text { Hip internal rotation in } 90^{\circ} \text { flexion }\left(^{\circ}\right)\end{array}$ & $\begin{array}{l}28.5 \pm N R \dagger \\
28.9 \pm N R^{*} ף \\
113.7 \pm N R^{*} \\
16.7 \pm N R^{*} \emptyset\end{array}$ & $\begin{array}{l}34.1 \pm \mathrm{NR} \\
38.4 \pm \mathrm{NR} \\
125.0 \pm \mathrm{NR} \\
28.0 \pm \mathrm{NR}\end{array}$ & $\begin{array}{l}32.5 \pm N R \\
38.0 \pm N R \\
120.8 \pm N R \\
27.8 \pm N R\end{array}$ \\
\hline Audenaert, 2012b & ROM & CT modelling & $\begin{array}{l}\text { Range of hip internal rotation in high flex }\left(^{\circ}\right) \\
\text { Range of hip internal rotation on impingement test } \\
\left(^{\circ}\right)\end{array}$ & $\begin{array}{l}12.9 \pm 6.4 * \emptyset \\
12.3 \pm 6.5 * \emptyset\end{array}$ & $\begin{array}{l}27.8 \pm 7.6 \\
27.9 \pm 7.4\end{array}$ & $\begin{array}{l}20.9 \pm 9.1^{*} \\
21.1 \pm 8.8^{*}\end{array}$ \\
\hline Brisson, 2013 & $\begin{array}{l}\text { ROM } \\
\text { Joint Forces }\end{array}$ & 3-Dimensional motion analysis during gait & $\begin{array}{l}\text { Sagittal hip ROM }\left(^{\circ}\right) \\
\text { Frontal hip ROM }\left({ }^{\circ}\right) \\
\text { Peak hip flexion }(\mathrm{Nm} / \mathrm{kg}) \\
\text { Peak hip external rotation }(\mathrm{Nm} / \mathrm{kg})\end{array}$ & $\begin{array}{l}47.4 \pm 3.6 \dagger \\
14.3 \pm 2.6 \dagger \\
0.98 \pm 0.23 \\
0.11 \pm 0.04\end{array}$ & $\begin{array}{l}51.5 \pm 2.7 \\
17.8 \pm 2.9 \\
1.05 \pm 0.31 \\
0.12 \pm 0.03\end{array}$ & \\
\hline Hunt, 2013 & $\begin{array}{l}\text { ROM } \\
\text { Joint Forces }\end{array}$ & 3-Dimensional motion analysis during gait & $\begin{array}{l}\text { Peak hip extension }\left(^{\circ}\right) \\
\text { Peak hip adduction }\left(^{\circ}\right) \\
\text { Peak hip internal rotation }\left(^{\circ}\right) \\
\text { Peak hip flexion }(\mathrm{Nm} / \mathrm{kg}) \\
\text { Peak hip external rotation }(\mathrm{Nm} / \mathrm{kg})\end{array}$ & $\begin{array}{l}7.4 \pm 6.7 \dagger \\
4.1 \pm 3.7 \dagger \\
3.1 \pm 4.2 \dagger \\
0.48 \pm 0.15 \dagger \\
0.12 \pm 0.04 \dagger\end{array}$ & $\begin{array}{l}12.0 \pm 7.2 \\
5.7 \pm 2.9 \\
8.2 \pm 5.8 \\
0.56 \pm 0.16 \\
0.15 \pm 0.03\end{array}$ & \\
\hline Rylander, 2013 & ROM & $\begin{array}{l}\text { 3-Dimensional motion analysis during stair } \\
\text { climbing }\end{array}$ & $\begin{array}{l}\text { Sagittal hip ROM }\left(^{\circ}\right) \\
\text { Hip rotation ROM }\left(^{\circ}\right) \\
\text { Max hip internal rotation }\left(^{\circ}\right) \\
\text { Frontal hip ROM }\left(^{\circ}\right) \\
\text { Max hip abduction }\left(^{\circ}\right) \\
\text { Sagittal hip ROM }\left(^{\circ}\right) \\
\text { Max hip extension }\left(^{\circ}\right) \\
\text { Max hip internal rotation }\left(^{\circ}\right) \\
\text { Pelvic rotation ROM }\left(^{\circ}\right) \\
\text { Max pelvic tilt }\left(^{\circ}\right)\end{array}$ & $\begin{array}{l}40.0 \pm 5.7 \dagger \\
11.3 \pm 3.5 \dagger \\
6.5 \pm 4.6 \dagger \\
10.0 \pm 2.2^{*} \\
(-) 1.1 \pm 5.0 \dagger \\
54.8 \pm 3.7^{*} \\
(-) 11.4 \pm 6.9 \dagger \\
7.1 \pm 6.4 \dagger \\
13.8 \pm 6.3^{*} \\
20.8 \pm 6.2^{*}\end{array}$ & $\begin{array}{l}44.1 \pm 4.8 \\
14.0 \pm 4.4 \\
11.0 \pm 5.4 \\
13.4 \pm 4.1 \\
2.2 \pm 3.3 \\
60.0 \pm 4.5 \\
(-) 6.6 \pm 4.0 \\
12.1 \pm 4.2 \\
8.3 \pm 4.9 \\
14.3 \pm 3.9\end{array}$ & \\
\hline
\end{tabular}

*Significant difference $p<0.01$ compared to asymptomatic with no FAl.

tSignificant difference $p<0.05$ compared to asymptomatic with no FAI.

$\ddagger=$ Contralateral limb of FAl group.

$\S=$ Not tested for significance.

Significant difference $p<0.01$ compared to asymptomatic with FAI.

FAl, femoroacetabular impingement; NR, not reported; TFL, tensor fasciae latae; RF, rectus femoris; ROM, range of motion. 
Neuromuscular activity_compared to controls with uncertain hip morphology

Two studies examined muscle activation levels of rectus femoris and tensor fasciae latae. ${ }^{41}{ }^{42}$ One reported significantly decreased absolute tensor fasciae latae activity level in symptomatic FAI compared with control participants during maximal active hip flexion contraction, but no difference for rectus femoris. ${ }^{41}$ The second study found no differences for either muscle group when relative EMG activity was measured during submaximal isometric fatigue testing. ${ }^{42}$

Activity limitation—squatting ability_compared to controls with no FAl hip morphology

One study reported that people with symptomatic FAI were unable to squat as deeply as a control group. ${ }^{30}$

\section{Interventional studies evaluating effects of treatment on physical impairments and/or activity limitations}

Main findings of intervention studies are summarised in table 4. All five were observational within-subjects study designs. Four studies evaluated hip arthroscopy and one evaluated a conservative treatment programme. Follow-up varied between 8 and 32 months. Methodological quality was moderate to high, with scores ranging between 7 and 8 stars (figure 2). All included intervention studies were a level $3 \mathrm{~b}$ of evidence (figure 2). The only impairment evaluated was ROM; the only measured activity limitation was squatting ability. A meta-analysis was not performed because of small study numbers and high heterogeneity in outcome measures.

\section{Range of motion}

One study examined hip ROM 25-28 months after a four-stage conservative treatment programme comprised of avoidance of excessive physical activity and anti-inflammatory drugs, physiotherapy and modifications of activities of daily living. No significant changes in hip ROM were reported following treatment. Furthermore, hip ROM remained less than the contralateral hip without FAI at follow-up. ${ }^{18}$

Three studies examined the effects of hip arthroscopy on hip ROM during gait using three-dimensional motion analysis. ${ }^{33} 3538$ Twelve months following surgery one study reported a significant increase in sagittal hip ROM during stance. ${ }^{38}$ Another study reported similar findings, plus postoperative increase in hip rotation ROM and maximal hip internal rotation. ${ }^{35}$ The third study reported no significant change in sagittal hip ROM at $10-32$ months after surgery, ${ }^{33}$ and reported ROM in symptomatic FAI remained significantly less than controls (table 3).

One study examined the effects of arthroscopy on hip ROM during stair climbing and found no differences at 12 months compared with preoperative values, ${ }^{35}$ and further indicated ROM remained impaired compared with controls who were not imaged to ensure no evidence of FAI (table 3). ${ }^{35}$

Arthroscopy had no significant effect on hip joint ROM during squatting at $8-32$ months postsurgery measured using threedimensional motion analysis compared with preoperative values. ${ }^{39}$

\section{Activity limitation-squatting ability}

Squatting ability (measured as increased mean maximum squat depth as a percentage of leg length) was significantly improved at $8-32$ months postarthroscopy compared with preoperative ability. ${ }^{39}$

\section{DISCUSSION}

In this systematic review, we included 16 studies (14 crosssectional comparisons-level 4 evidence, five intervention studies-level $3 \mathrm{~b}$ evidence) to establish whether people with symptomatic FAI demonstrate physical impairments and/or activity limitations compared with people without FAI and whether treatment improved these parameters. We found that people with symptomatic FAI have some physical impairments (mainly in the domain of reduced ROM) and limited evidence of activity limitations when compared with individuals without FAI. We found that arthroscopy does not necessarily improve hip ROM.

Most FAI research has focused on ROM impairments and suggest that individuals with symptomatic FAI have decreased hip ROM towards impingement (flexion/internal rotation in $90^{\circ}$ flexion). ${ }^{28} 31323637$ Pain likely plays a role since people with symptomatic FAI demonstrate reduced ROM compared with people with asymptomatic FAI. ${ }^{32}$ However, people with asymptomatic FAI also demonstrate reduced ROM compared with people with no evidence of FAI, suggesting that pain is not solely responsible. ${ }^{32}$ Impaired ROM may be partially explained by the bony impingement/abutment, as emphasised by the reduced hip ROM reported using CT modelling, ${ }^{37}$ and/or damage to the surrounding soft tissue.

Some evidence suggests that hip ROM during walking is reduced in people with symptomatic FAI, despite the fact that motion in this task does not reach the end of available range. ${ }^{43}$ Although four studies reported reduced ROM in the frontal plane, ${ }^{29} 33-35$ only two of them imaged control participants to ensure no evidence of underlying asymptomatic FAI. ${ }^{29} 33$ Inconsistencies were evident in sagittal plane findings regardless of control group imaging. Furthermore, the clinical significance of these small, albeit significant, reported differences in hip ROM are not known, but are possibly of little clinical relevance.

Most studies did not demonstrate differences in peak hip joint moments compared with controls. ${ }^{29} 33$ While one study did report differences, ${ }^{34}$ these must be interpreted with caution since no imaging was used to ensure that controls did not have underlying FAI morphology.

One study each evaluated strength and fatigue in $\mathrm{FAI}^{41} 42$ and suggested that strength deficits ( $75 \%$ of control strength), particularly of the hip flexor and adductor muscles, are present in people with symptomatic FAI compared with controls. However, controls were not imaged to ensure absence of FAI and this might account for similar between group findings in other planes; some literature places the likelihood of asymptomatic FAI in healthy young adults as high as $35 \% .{ }^{44}$ Further studies are required to investigate strength in people with symptomatic FAI across all movement directions and should include asymptomatic control groups that have been imaged to ensure absence of FAI. There is also limited evidence that people with symptomatic FAI exhibited decreased absolute tensor fasciae lata EMG activity during maximal active hip flexion contraction. ${ }^{41}$ However, most studies normalise absolute EMG to a reference such as a maximum voluntary contraction due to the influence of such things as subcutaneous fat on the absolute EMG signal. $^{45}$ Future studies should examine relative muscle activation levels between muscles during functional tasks compared with an appropriately imaged control group, given the important role that these muscles are likely to play in augmenting hip stability. ${ }^{46}$

Only five intervention studies were included in this review: four described the outcomes of hip arthroscopy and one evaluated conservative management. Hip arthroscopy appears to improve hip ROM in the sagittal, but not frontal plane during gait. $^{35} 38$ Reduced hip ROM during stair climbing remained following surgical intervention despite correction/reduction of the 


\begin{tabular}{|c|c|c|c|c|c|c|c|c|c|}
\hline \multicolumn{10}{|c|}{ Intervention studies } \\
\hline Author, Year & $\begin{array}{l}\text { Impairment and/or } \\
\text { activity limitation } \\
\text { investigated }\end{array}$ & Measurement method & $\begin{array}{l}\text { Variables } \\
\text { measured }\end{array}$ & $\begin{array}{l}\text { Preintervention } \\
\text { score }(m e a n \pm S D)\end{array}$ & $\begin{array}{l}\text { Postintervention } \\
\text { score (mean } \pm S D \text { ) }\end{array}$ & Effect sizes $(95 \% \mathrm{Cl})$ & Intervention & Follow-up time & Intervention details \\
\hline \multirow[t]{3}{*}{ Lamontagne, 2011} & Squatting ability & \multirow[t]{3}{*}{$\begin{array}{l}\text { Three-dimensional motion } \\
\text { analysis during squatting }\end{array}$} & $\begin{array}{l}\text { Mean max squat } \\
\text { depth (\%) }\end{array}$ & $36.9 \pm 12.0$ & $33.2 \pm 10.3^{*}$ & $-0.32(-1.20$ to 0.56$)$ & \multirow[t]{3}{*}{ Arthroscopy } & \multirow[t]{3}{*}{$\begin{array}{l}\text { 8-32 months } \\
\text { postarthroscopy }\end{array}$} & Open or combined approach \\
\hline & \multirow[t]{2}{*}{ ROM } & & $\begin{array}{l}\text { Sagittal hip } \\
\text { angles }\left(^{\circ}\right)\end{array}$ & $109.8 \pm 9.7$ & $106.2 \pm 8.8$ & $-0.37(-1.26$ to 0.51$)$ & & & \multirow[t]{2}{*}{$\begin{array}{l}\text { Dislocation of the hip and debridement } \\
\text { of the proximal femur }\end{array}$} \\
\hline & & & $\begin{array}{l}\text { Sum of all joint } \\
\text { angles (hip, knee, } \\
\text { ankle) ( }\left(^{\circ}\right)\end{array}$ & $263.2 \pm 33.1$ & $277.9 \pm 35.5^{*}$ & $0.41(-0.48$ to 1.30$)$ & & & \\
\hline \multirow[t]{8}{*}{ Emara, 2011} & \multirow[t]{8}{*}{ ROM } & \multirow[t]{8}{*}{ Not reported } & Hip flexion $\left(^{\circ}\right)$ & $95.0 \pm 0.4$ & $+88.0 \pm 3.5$ & $-3.55(-4.28$ to $(-2.82))$ & \multirow{8}{*}{$\begin{array}{l}\text { Conservative } \\
\text { treatment } \\
\text { programme }\end{array}$} & \multirow{8}{*}{$\begin{array}{l}\text { Every } 6 \text { months for } \\
24 \text { months }\end{array}$} & 4 Stages of conservative treatment \\
\hline & & & Hip extension $\left({ }^{\circ}\right)$ & $4.0 \pm 1.6$ & $+3.6 \pm 2.2$ & $-0.21(-0.67$ to 0.25$)$ & & & $\begin{array}{l}\text { 1. Avoidance of excessive phys. activity } \\
\text { and drugs ( } 2-4 \text { weeks) }\end{array}$ \\
\hline & & & Hip abduction $\left({ }^{\circ}\right)$ & $37.0 \pm 0.4$ & $\dagger 36.0 \pm 1.4$ & $-1.10(-1.59$ to $(-0.61))$ & & & $\begin{array}{l}\text { 2. Physiotherapy ( } 2-3 \text { weeks stretching } \\
20-30 \text { min daily) }\end{array}$ \\
\hline & & & Hip adduction $\left({ }^{\circ}\right)$ & $17.0 \pm 7.0$ & $\dagger 17.0 \pm 9.0$ & $0.00(-0.46$ to 0.46$)$ & & & $\begin{array}{l}\text { 3. Assessment of normal ROM after acute } \\
\text { pain subsided }\end{array}$ \\
\hline & & & $\begin{array}{l}\text { Hip external } \\
\text { rotation in } \\
\text { flexion }\left(^{\circ}\right)\end{array}$ & $28.5 \pm 0.5$ & $\dagger 27.0 \pm 1.1$ & $-1.86(-2.40$ to $(-1.31))$ & & & $\begin{array}{l}\text { 4. Modification of activities of daily living } \\
\text { predisposing to FAI }\end{array}$ \\
\hline & & & $\begin{array}{l}\text { Hip external } \\
\text { rotation in } \\
\text { extension }\left(^{\circ}\right)\end{array}$ & $25.3 \pm 0.3$ & $\dagger 24.6 \pm 1.0$ & $-1.07(-1.55$ to $(-0.58))$ & & & $\begin{array}{l}\text { Follow-ups every 2-3 weeks until } \\
\text { symptoms resolved, then every } 3 \text { mo. for } \\
12 \text { months, and every } 6 \text { mo. thereafter }\end{array}$ \\
\hline & & & $\begin{array}{l}\text { Hip internal } \\
\text { rotation in } \\
\text { flexion }\left(^{\circ}\right)\end{array}$ & $9.4 \pm 0.3$ & $\dagger 10.0 \pm 0.6$ & 1.32 (0.82 to 1.82$)$ & & & \\
\hline & & & $\begin{array}{l}\text { Hip internal } \\
\text { rotation in } \\
\text { extension }\left(^{\circ}\right)\end{array}$ & $15.8 \pm 0.4$ & $\dagger 15.8 \pm 0.7$ & $0.00(-0.46$ to 0.46$)$ & & & $\begin{array}{l}\text { Surgery indicated when conservative } \\
\text { treatment failed }\end{array}$ \\
\hline \multirow[t]{2}{*}{ Rylander, 2012} & \multirow[t]{2}{*}{ ROM } & \multirow[t]{2}{*}{$\begin{array}{l}\text { Three-dimensional motion } \\
\text { analysis during gait }\end{array}$} & $\begin{array}{l}\text { Sagittal hip } \\
\text { ROM }\left({ }^{\circ}\right)\end{array}$ & $27.6 \pm 5.0$ & $30.7 \pm 4.3^{*}$ & $0.64(-0.22$ to 1.50$)$ & \multirow[t]{2}{*}{ Arthroscopy } & \multirow[t]{2}{*}{$\begin{array}{l}12 \text { month } \\
\text { postarthroscopy }\end{array}$} & \multirow[t]{2}{*}{ Arthroplastic reshaping surgery } \\
\hline & & & Max hip flexion $\left(^{\circ}\right)$ & $19.9 \pm \mathrm{NR}$ & $22.7 \pm N R^{*}$ & unable to be determined & & & \\
\hline \multirow[t]{2}{*}{ Brisson, 2013} & \multirow[t]{2}{*}{ ROM } & \multirow[t]{2}{*}{$\begin{array}{l}\text { Three-dimensional motion } \\
\text { analysis during gait }\end{array}$} & $\begin{array}{l}\text { Sagittal hip } \\
\text { ROM }\left(^{\circ}\right)\end{array}$ & $47.4 \pm 3.6$ & $46.8 \pm 4.6$ & $-0.14(-1.02$ to 0.74$)$ & \multirow[t]{2}{*}{ Arthroscopy } & \multirow[t]{2}{*}{$\begin{array}{l}\text { 10-32 months } \\
\text { postarthroscopy }\end{array}$} & $\begin{array}{l}\text { Open or combined approach } \\
\text { Dislocation of the hip and debridement } \\
\text { of the proximal femur }\end{array}$ \\
\hline & & & $\begin{array}{l}\text { Frontal hip } \\
\text { ROM }\left(^{\circ}\right)\end{array}$ & $14.3 \pm 2.6$ & $14.3 \pm 2.7$ & $0.00(-0.88$ to 0.88$)$ & & & $\begin{array}{l}\text { Standard post-operative rehab } \\
\text { programme } \\
\text { recommended by Healthcare professional }\end{array}$ \\
\hline \multirow[t]{4}{*}{ Rylander, 2013} & \multirow[t]{4}{*}{ ROM } & \multirow{4}{*}{$\begin{array}{l}\text { Three-dimensional motion } \\
\text { analysis during gait and stair } \\
\text { climbing }\end{array}$} & $\begin{array}{l}\text { Sagittal hip ROM } \\
\text { (॰) (gait) }\end{array}$ & $40.0 \pm 5.7$ & $42.5 \pm 7.5^{*}$ & $0.37(-0.44$ to 1.17$)$ & \multirow[t]{4}{*}{ Arthroscopy } & \multirow[t]{4}{*}{$\begin{array}{l}12 \text { month } \\
\text { postarthroscopy }\end{array}$} & \multirow[t]{4}{*}{$\begin{array}{l}\text { Arthroscopic acetabuloplasty and/or } \\
\text { cheilectomy }\end{array}$} \\
\hline & & & $\begin{array}{l}\text { Max hip flexion }\left({ }^{\circ}\right) \\
\text { (gait) }\end{array}$ & $35.5 \pm 5.3$ & $37.5 \pm 5.9^{*}$ & 0.34 (-0.46 to 1.15$)$ & & & \\
\hline & & & $\begin{array}{l}\text { Hip rotation ROM } \\
\text { (०) (gait) }^{\text {(g) }}\end{array}$ & $11.3 \pm 3.5$ & $14.1 \pm 5.1 \neq$ & $0.63(-0.19$ to 1.45$)$ & & & \\
\hline & & & $\begin{array}{l}\text { Max hip int. } \\
\text { rotation }\left(^{\circ} \text { ) (gait) }\right.\end{array}$ & $6.5 \pm 4.6$ & $10.3 \pm 5.6^{*}$ & $0.72(-0.11$ to 1.55$)$ & & & \\
\hline
\end{tabular}


morphological abnormalities, ${ }^{35}$ suggesting that hip function in the sagittal or transverse planes may not resolve spontaneously during activities that require greater hip ROM. Thus, although surgical intervention may restore range of hip motion, training may be required for patients to use the additional range in function. A major conclusion from our review is the urgent need to evaluate the role of rehabilitation programmes targeted at postoperative restoration of functional impairments in people with symptomatic FAI.

One study evaluated the effects of conservative intervention on physical impairments associated with symptomatic FAI. ${ }^{18}$ Treatment comprised avoidance of excessive physical activity and anti-inflammatory drugs, physiotherapy and modifications of activities of daily living, and had no effect on hip ROM, which remained less than the contralateral limb without FAI. The study was limited by its short period of poorly-defined physiotherapy and no documentation of adherence. Further trials should investigate postoperative rehabilitation programmes and other options for conservative treatment.

Although there is limited literature evaluating activity limitations in people with symptomatic FAI, one study reported that people with symptomatic FAI were unable to squat as deeply as controls. ${ }^{30}$ This raises important clinical implications given the likely relationship between this task and functional tasks (including sport activities) that involve a similar range of hip flexion with concurrent demands on balance and stability. Squatting limitation may be a function of hip morphology or pain/avoidance strategies. Hip arthroscopy can increase squat depth within 8-32 months postoperatively, ${ }^{39}$ restoring it to a depth resembling that of healthy controls. ${ }^{30}$ Further research of activities that involve positions of impingement paralleling those required in sport (eg, step-up tasks, kicking) would help inform the impact of symptomatic FAI and the effect of treatment (surgical and conservative).

This review was limited to 16 articles, including some with conflicting observations. The variability in how FAI was diagnosed across studies makes it difficult to draw firm conclusions about the impact of FAI on physical impairments, as these may vary according to diagnostic criteria. A meta-analysis was not possible because of the small number of available studies, and the high heterogeneity of methods and outcome measures. Many studies had relatively small sample sizes that were not necessarily supported by a priori sample size calculations. A further limitation is the inclusion of five studies ${ }^{34} 3540-42$ that used a control group without imaging to ensure no evidence of FAI. It is possible that some control participants in these studies may have had underlying asymptomatic FAI, thus we must be prudent when interpreting these findings.

The strengths of this review include the reproducible search strategy, application of the PRISMA checklist for reporting of findings ${ }^{20}$ and use of a quality assessment tool. $^{22} 23$ This review is limited by an English-only search strategy, the paucity of studies in this area, the heterogeneity in study methods and outcomes, and the inconsistency in reported results. No studies included treatment evidence from randomised control trials.

A greater understanding of physical impairments and activity limitations is required before the effects of treatments can be fully evaluated. This should be a priority for future research, as should be ensuring patient reported outcome measures are used to better understand activity limitations. Future investigations should examine physical impairments and activity limitations in dynamic tasks that replicate sporting tasks-designed to target positions of impingement. This will establish a baseline to compare the effectiveness of treatments (surgical and conservative), and assist in the design of conservative management programmes, including postoperative rehabilitation. Subsequently, future randomised control trials can examine treatment effects on FAI and, importantly, consider whether non-invasive treatments are a viable and/or preferred alternative to surgical intervention.

Although all studies scored moderately well on the NOS for quality assessment, the five studies with lowest scores (5-6/9) were those that did not image control group participants to ensure no evidence of FAI. Reported differences in all studies must be interpreted with caution since groups were selected from different communities, making it impossible to exclude factor(s) other than FAI. Future cross-sectional studies should ensure that control groups are matched on all key criteria such as age, gender, body mass index and activity level. Further, a consistent definition of FAI should be applied in future studies, and imaging of control participants is necessary to exclude asymptomatic FAI and ensure findings are purely a function of disease.

\section{CONCLUSION}

People with symptomatic FAI have physical impairments in hip ROM, particularly into directions of hip impingement. There is suggestion of impairments in hip frontal, sagittal and transverse plane ROM during gait, squatting and stair climbing, although evidence is only at a 3b-4 level. Little attention has been directed towards activity limitations in symptomatic FAI and limited research demonstrates reduced squatting ability. Arthroscopy may improve some, but not all, impairments in ROM. Physiotherapy may be needed to normalise ROM after hip arthroscopy. Further research is needed to: (1) determine whether hip ROM and neuromuscular function are compromised in dynamic tasks designed to target positions of impingement in FAI; and (2) to evaluate the effects of non-surgical treatments, such as joint mobilisation techniques, hip bracing and targeted exercise programmes incorporating ROM, strengthening and/or neuromuscular retraining, on physical impairments and activity limitations.

Acknowledgements The authors acknowledge the valuable contribution of Philippa Nicolson in this work.

Contributors LED, TVW, PWH, KLB and RSH conceived the idea for the paper. LED and FLD performed the literature search. LED wrote the first draft of the article. All authors revised the paper and provided scientific input. All authors approved the final version of the manuscript. RSH is the guarantor (the contributor who accepts full responsibility for the finished article, had access to any data and controlled the decision to publish).

Funding Funding was provided by a Program Grant from the National Health and Medical Research Council (NHMRC) of Australia (ID631717). KLB is supported by a Future Fellowship from the Australian Research Council (FT0991413). PWH is supported by a Senior Principal Research Fellowship (APP1002190) from the NHMRC. RSH is supported by an Australian Research Council Future Fellowship (FT130100175).

Competing interests KLB reports grants from NHMRC Program Grant, during the conduct of the study; other from Gel Melbourne OA shoe sales, other from

Educational OA DVD for physios, grants from ARC and NHMRC grants, outside the submitted work. TVW reports and ASICS Oceania Pty Ltd possible future royalties on shoe sales (ie, none as yet). PWH reports grants from National Health and Medical Research Council of Australia, outside the submitted work. RSH reports grants from NHMRC Program Grant, during the conduct of the study; other from Gel Melbourne OA shoe sales, other from Educational OA DVD for physios, grants from ARC and NHMRC grants, outside the submitted work.

Ethics approval Behavioural and Social Sciences Human Ethics Sub-CommitteeThe University of Melbourne.

Provenance and peer review Not commissioned; externally peer reviewed. 


\section{REFERENCES}

1 Sierra RJ, Trousdale RT, Ganz R, et al. Hip disease in the young, active patient: evaluation and nonarthroplasty surgical options. J Am Acad Orthop Surg 2008;16:689-703.

2 Cheatham S, Kolber M. Rehabilitation after hip arthroscopy and labral repair in a high school football athlete. Int J Sports Phy Ther 2012;7:173-84.

3 Ganz R, Parvizi J, Beck M, et al. Femoroacetabular impingement: a cause for osteoarthritis of the hip. Clin Orthop Relat Res 2003;417:112-20.

4 Beck $M$, Kalhor $M$, Leunig $M$, et al. Hip morphology influences the pattern of damage to the acetabular cartilage: femoroacetabular impingement as a cause of early osteoarthritis of the hip. J Bone Joint Surg Am 2005;87:1012-18.

5 Philippon MJ, Schenker ML. Athletic hip injuries and capsular laxity. Operative Tech Orthop 2005;15:261-6.

6 Leunig M, Beaule PE, Ganz R. The concept of femoroacetabular impingement: current status and future perspectives. Clin Orthop Relat Res 2009;467:616-22.

7 Philippon MJ, Maxwell RB, Johnston TL, et al. Clinical presentation of femoroacetabular impingement. Knee Surg Sports Traumatol Arthrosc 2007; 15:1041-7.

8 Beck M, Leunig M, Parvizi J, et al. Anterior femoroacetabular impingement: part II. Midterm results of surgical treatment. Clin Orthop Relat Res 2004;418:67-73.

9 Boykin R, Stull J, Giphart JE, et al. Femoroacetabular impingement in a professional soccer player. Knee Surg Sports Traumatol Arthrosc 2013;21:1203-11.

10 Philippon M, Schenker M, Briggs K, et al. Femoroacetabular impingement in 45 professional athletes: associated pathologies and return to sport following arthroscopic decompression. Knee Surg Sports Traumatol Arthrosc 2007;15:908-14.

11 Kemp JL, Makdissi M, Schache AG, et al. Hip chondropathy at arthroscopy: prevalence and relationship to labral pathology, femoroacetabular impingement and patient-reported outcomes. Br J Sports Med 2014;48:1102-7.

12 Agricola R, Heijboer MP, Bierma-Zeinstra SM, et al. Cam impingement causes osteoarthritis of the hip: a nationwide prospective cohort study (CHECK). Ann Rheum Dis 2013;72:918-23.

13 Philippon M, Schenker M. Arthroscopy for the treatment of femoroacetabular impingement in the athlete. Clin Sports Med 2006;25:299-308, ix.

14 Clohisy JC, St John LC, Schutz AL. Surgical treatment of femoroacetabular impingement: a systematic review of the literature. Clin Orthop Relat Res 2010;468:555-64.

15 Stevens $M$, Legay $D$, Glazebrook $M$, et al. The evidence for hip arthroscopy: grading the current indications. Arthroscopy 2010;26:1370-83.

$16 \mathrm{Ng} \mathrm{V}$, Arora N, Best T, et al. Efficacy of surgery for femoroacetabular impingement: a systematic review. Am J Sports Med 2010;38:2337-45.

17 Kemp JL, Collins NJ, Makdissi M, et al. Hip arthroscopy for intra-articular pathology: a systematic review of outcomes with and without femoral osteoplasty. $\mathrm{Br} J$ Sports Med 2012;46:632-43.

18 Emara K, Samir W, Motasem el H, et al. Conservative treatment for mild femoroacetabular impingement. J Orthop Surg (Hong Kong) 2011;19:41-5.

19 Wall PD, Fernandez M, Griffin D, et al. Nonoperative treatment for femoroacetabular impingement: a systematic review of the literature. PM R 2013;5:418-26.

20 Moher D, Liberati A, Tetzlaff J, et al. Preferred reporting items for systematic reviews and meta-analyses: the PRISMA statement. Int J Surg 2010;8:336-41.

21 World Health Organization. International Classification of Functioning, Disability and Health (ICF). Geneva: World Health Organization, 2013. [updated 2013; cited 25 Nov 2013]. http://www.who.int/classifications/icf/en/

22 Wells GA, Shea B, O'Connell D, et al. The Newcastle-Ottawa Scale (NOS) for assessing the quality of nonrandomized studies in meta-analyses. [cited $14 \mathrm{Mar}$ 2013]. http://www.ohri.ca/programs/clinical_epidemiology/oxford.asp

23 Li W, Ma D, Liu M, et al. Association between metabolic syndrome and risk of stroke: a meta-analysis of cohort studies. Cerebrovasc Dis 2008;25:539-47.

24 Stang A. Critical evaluation of the Newcastle-Ottawa scale for the assessment of the quality of nonrandomized studies in meta-analyses. Eur J Epidemiol 2010:25:603-5.
25 Centre for Evidence Based Medicine. Oxford Centre for Evidence-based MedicineLevels of Evidence (March 2009). University of Oxford, 2009. [cited 1 Apr 2014]. http://www.cebm.net/?0=1025

26 Lakens D. Calculating and reporting effect sizes to facilitate cumulative science: a practical primer for -tests and ANOVAs. Front Psychol 2013:4:863.

27 Cumming G, Finch S. Inference by eye: confidence intervals and how to read pictures of data. Am Psychol 2005;60:170-80.

28 Kennedy $M$, Lamontagne $M$, Beaulé $P$. The effect of cam femoroacetabular impingement on hip maximal dynamic range of motion. J Orthop 2009:1:45-50.

29 Kennedy MJ, Lamontagne M, Beaule PE. Femoroacetabular impingement alters hip and pelvic biomechanics during gait Walking biomechanics of FAl. Gait Posture 2009;30:41-4.

30 Lamontagne M, Kennedy MJ, Beaule PE. The effect of cam FAI on hip and pelvic motion during maximum squat. Clin Orthop Relat Res 2009;467:645-50.

31 Audenaert E, Van Houcke J, Maes B, et al. Range of motion in femoroacetabular impingement. Acta Orthop Belg 2012;78:327-32.

32 Audenaert EA, Peeters I, Vigneron L, et al. Hip morphological characteristics and range of internal rotation in femoroacetabular impingement. Am J Sports Med 2012;40:1329-36.

33 Brisson N, Lamontagne M, Kennedy MJ, et al. The effects of cam femoroacetabular impingement corrective surgery on lower-extremity gait biomechanics. Gait Posture 2013;37:258-63

34 Hunt MA, Gunether JR, Gilbart MK. Kinematic and kinetic differences during walking in patients with and without symptomatic femoroacetabular impingement. Clin Biomech (Bristol, Avon) 2013;28:519-23.

35 Rylander J, Shu B, Favre J, et al. Functional testing provides unique insights into the pathomechanics of femoroacetabular impingement and an objective basis for evaluating treatment outcome. J Orthop Res 2013;31:1461-8.

36 Kubiak-Langer M, Tannast M, Murphy SB, et al. Range of motion in anterior femoroacetabular impingement. Clin Orthop Relat Res 2007;458:117-24.

37 Tannast M, Kubiak-Langer M, Langlotz F, et al. Noninvasive three-dimensional assessment of femoroacetabular impingement. J Orthop Res 2007;25:122-31.

38 Rylander JH, Shu B, Andriacchi TP, et al. Preoperative and postoperative sagittal plane hip kinematics in patients with femoroacetabular impingement during level walking. Am J Sports Med 2011;39(Suppl):36S-42S

39 Lamontagne $\mathrm{M}$, Brisson N, Kennedy MJ, et al. Preoperative and postoperative lower-extremity joint and pelvic kinematics during maximal squatting of patients with cam femoro-acetabular impingement. J Bone Joint Surg Am 2011;93(Suppl 2):40-5.

40 Nussbaumer S, Leunig M, Glatthorn JF, et al. Validity and test-retest reliability of manual goniometers for measuring passive hip range of motion in femoroacetabular impingement patients. BMC Musculoskelet Disord 2010;11:194

41 Casartelli NC, Maffiuletti NA, Item-Glatthorn JF, et al. Hip muscle weakness in patients with symptomatic femoroacetabular impingement. Osteoarthritis Cartilage 2011;19:816-21.

42 Casartelli NC, Leunig M, Item-Glatthorn JF, et al. Hip flexor muscle fatigue in patients with symptomatic femoroacetabular impingement. Int Orthop 2012;36:967-73

43 Neumann D. Kinesiology of the musculoskeletal system: foundations for physical rehabilitation. Mosby, Inc., 2002.

44 Laborie LB, Lehmann TG, Engesæter I, et al. Prevalence of radiographic findings thought to be associated with femoroacetabular impingement in a population-based cohort of 2081 healthy young adults. Radiology 2011;260:494-502.

45 Burden AM, Trew M, Baltzopoulos V. Normalisation of gait EMGs: a re-examination. J Electromyogr Kinesiol 2003;13:519-32.

46 Retchford TH, Crossley KM, Grimaldi A, et al. Can local muscles augment stability in the hip? A narrative literature review. J Musculoskelet Neuronal Interact 2013;13:1-12. 